\title{
MONOTONICITY OF FUNCTIONALS ALONG CONFORMAL RICCI FLOW
}

\author{
FENGJIANG LI, PENG LU, JIANHONG WANG*, AND YU ZHENG
}

\begin{abstract}
The main purpose of this note is to construct two functionals of the positive solutions to the conjugate heat equation associated to the metrics evolving by the conformal Ricci flow on closed manifolds. We show that they are nondecreasing by calculating the explicit evolution formulas of these functionals. For the entropy functional we give another proof of the monotonicity by establishing a pointwise formula. Moreover, we show that the increase are strict unless the metrics are Einstein.
\end{abstract}

\section{INTRODUCTION}

Monotonicity formulas (geometric quantities which are monotone along geometric flows) play a key role in geometry and analysis, when being used deliberately they give various kind geometric information including rigidity results. Two outstanding examples are Huisken's monotonicity formula for the mean curvature flow [Hui90 and Perelman's $\mathcal{W}$-entropy for the Ricci flow Per02]. Inspired by the work [EKNT08, and [Mul10, a general approach to find monotone quantities on manifolds with evolving metrics is developed by H.-X. Guo, R. Philipowski, and A. Thalmaier in GPT13.

The conformal Ricci flow (CRF in short) is proposed by A. Fischer [Fis04] and is further studied in, for example, [LQZ14], SSZ18], and [LQZ19]. Going into a little details CRF considers a family of metrics $g=g(t), t \in[0, T)$, on an $(m+1)$-dimensional closed manifold $M$, which is evolved by

$$
\begin{cases}\partial_{t} g=-2\left(\mathrm{Rc}_{g(t)}+(p(t)+m) g(t)\right) & \text { on } M \times[0, T), \\ \left(-\Delta_{g(t)}+(m+1)\right) p(t)=\frac{1}{m}\left|\mathrm{Rc}_{g(t)}+m g(t)\right|^{2} & \text { on } M \times[0, T), \\ g(0)=g_{0}, & \end{cases}
$$

where Rc is the Ricci curvature, $p(t)$ is a scalar function, and $T$ is a positive constant. We assume that the initial metric $g_{0}$ has constant scalar curvature $-m(m+1)$.

Date: October 11, 2019.

2010 Mathematics Subject Classification. 53C44.

Key words and phrases. Conformal Ricci flow, conjugate heat equation, monotonicity of functionals.

* The corresponding author, email: jianhongwang@163.com. 
In this note, we apply the approach in GPT13 to CRF and find two new monotone quantities $\tilde{\mathcal{E}}(g, u)$ (see (2.6) for its definition and Lemma 2.1) and entropy $\tilde{\mathcal{W}}(g, u)$ defined by

$$
\tilde{\mathcal{W}}(g, u)=\int_{M}\left(|\nabla \ln u|^{2}+2(m+1) \ln u\right) u d \mu_{g}
$$

where $u$ is a positive function. More precisely we will consider the conjugate heat equation associated to $\mathrm{CRF}$

$$
\partial_{t} u=-\Delta_{g(t)} u+(m+1) p(t) u, \quad t \in[0, T) .
$$

It is easy to check that $\frac{d}{d t} \int_{M} u(t) d \mu_{g(t)}=0$. We will derive the following formula which is the main result of this note.

Theorem 1.1. Let $g(t)$ be a solution of $C R F$ (1.1) and let $u(x, t)$ be a positive solution of the conjugate heat equation (1.3). Define $\tilde{\mathcal{W}}(t)=\tilde{\mathcal{W}}(g(t), u(t))$. Then we have

$$
\begin{aligned}
\frac{d \tilde{\mathcal{W}}(t)}{d t}= & 2 \int_{M}|\mathrm{Rc}+m g-\nabla \nabla \ln u|^{2} u d \mu_{g(t)}+\frac{2}{m} \int_{M}|\mathrm{Rc}+m g|^{2} u d \mu_{g(t)} \\
& +2 \int_{M}|\nabla \ln u|^{2} p u d \mu_{g(t)}+2 \int_{M}|\nabla \ln u|^{2} u d \mu_{g(t)}
\end{aligned}
$$

for $t \in[0, T)$. Hence $\tilde{\mathcal{W}}(t)$ is nondecreasing, and the increase is strict unless $g(t)$ is an Einstein metric.

We will give two proofs of this theorem in $\S 2$ and $\S 3$, respectively. In the first proof we will see how the definition of $\tilde{\mathcal{W}}$ in (1.2) comes up. The second proof is a mimic of the proof of Perelman's entropy monotonicity given in [Per02, §9.1].

It should be pointed out that there is a long list of works on monotonicity formulas for various geometric flow (including static manifolds), many of them are inspired by the work in [Hui90] and [Per02]. We mention a few here as examples, L. Ni's entropy on static Riemannian manifolds [Ni04, List's entropy for extended Ricci flow Lis08, Müller's entropy for RicciHarmonic flow Mul12, and L.-F. Wang's entropy for Ricci-Bourguignon flow Wa19. The fancy part of Theorem 1.1 is that it is a monotonicity formula for coupled parabolic and elliptic equations.

\section{Proof of Theorem 1.1}

To facilitate our discussion of the derivation of (1.2), we now give a brief review of the general approach in GPT13. Let $\hat{M}^{n}$ be an n-dimensional closed manifold. We consider an abstract geometric flow of metrics $g(t)$ on $M$ defined by

$$
\partial_{t} g=-2 \alpha, \quad t \in[0, T),
$$


where $\alpha$ is a time-dependent symmetric two-tensor on $\hat{M}$. Let $A=\operatorname{tr}_{g} \alpha$. H.$\mathrm{X}$. Guo et al. construct the analog of Perelman's $\mathcal{F}$-energy and $\mathcal{W}$-entropy by defining

$$
\mathcal{F}(g, \phi)=\int_{\hat{M}}\left(A+|\nabla \phi|^{2}\right) e^{-\phi} d \mu_{g}
$$

for some function $\phi$, and

$$
\mathcal{W}(g, \psi, \tau)=\int_{\hat{M}}\left(\tau\left(A+|\nabla \psi|^{2}\right)+\psi-n\right)(4 \pi \tau)^{-\frac{n}{2}} e^{-\psi} d \mu_{g}
$$

for $\tau>0$ and function $\psi$ satisfying $\int_{\hat{M}}(4 \pi \tau)^{-\frac{n}{2}} e^{-\psi} d \mu_{g}=1$, respectively. They prove the following

Theorem A. (GPT13, Theorem 4.2 and 5.2]) (i) Suppose that $\phi$ satisfies the backward heat equation

$$
\partial_{t} \phi=-\Delta \phi+|\nabla \phi|^{2}-A, \quad t \in[0, T) .
$$

Then under the flow (2.1) the energy $\mathcal{F}(t)=\mathcal{F}(g(t), \phi(t))$ satisfies

$$
\frac{d \mathcal{F}(t)}{d t}=2 \int_{\hat{M}}\left(|\alpha+\nabla \nabla \phi|^{2}+\hat{\Theta}(-\nabla \phi)\right) e^{-\phi} d \mu_{g},
$$

where

$$
\hat{\Theta}(V)=(\operatorname{Rc}-\alpha)(V, V)+\langle\nabla A-2 \operatorname{Div}(\alpha), V\rangle+\frac{1}{2}\left(\partial_{t} A-2|\alpha|^{2}-\Delta A\right)
$$

for any vector field $V$. In particular, $\mathcal{F}(t)$ is nondecreasing when $\hat{\Theta} \geq 0$, and the increase is strict unless

$$
\alpha+\nabla \nabla \phi=0 \quad \text { and } \quad \hat{\Theta}(-\nabla \phi)=0 .
$$

(ii) Suppose that $\psi$ satisfies the backward heat equation

$$
\partial_{t} \psi=-\Delta \psi+|\nabla \psi|^{2}-A+\frac{n}{2 \tau}, \quad t \in[0, T),
$$

where $\tau=T-t$. Then under the flow (2.1) the entropy $\mathcal{W}(t)=\mathcal{W}(g(t), \psi(t)$, $T-t)$ satisfies

$$
\frac{d \mathcal{W}(t)}{d t}=\int_{\hat{M}} 2 \tau\left(\left|\alpha+\nabla \nabla \psi-\frac{1}{2 \tau} g\right|^{2}+\hat{\Theta}(-\nabla \psi)\right)(4 \pi \tau)^{-\frac{n}{2}} e^{-\psi} d \mu_{g} .
$$

In particular, $\mathcal{W}(t)$ is nondecreasing when $\hat{\Theta} \geq 0$, and the increase is strict unless

$$
\alpha+\nabla \nabla \psi-\frac{1}{2 \tau} g=0 \quad \text { and } \quad \hat{\Theta}(-\nabla \psi)=0 .
$$

On closed Riemannian manifold $\left(M^{m+1}, g\right)$, we define the BoltzmannShannon entropy $\tilde{\mathcal{E}}(g, u)$ by

$$
\tilde{\mathcal{E}}(g, u)=\int_{M} u \ln u d \mu_{g}
$$

where $u$ is a positive function. Towards the proof of Theorem 1.1 we start with the following 
Lemma 2.1. Let $g(t)$ be a solution of $C R F$ (1.1) and let $u(x, t)$ be a positive solution to the conjugate heat equation (1.3). Denote $\tilde{\mathcal{E}}(t)=\tilde{\mathcal{E}}(g(t), u(t))$, then we have

$$
\frac{d \tilde{\mathcal{E}}(t)}{d t}=\int_{M}\left(|\nabla \ln u|^{2}+(m+1) p\right) u d \mu_{g}
$$

Thus $\tilde{\mathcal{E}}(t)$ is nondecreasing and the increase is strict unless $g(t)$ is an Einstein metric.

Proof. By a direct computation or using [GPT13, Theorem 2.1] we have

$$
\begin{aligned}
\frac{d \tilde{\mathcal{E}}(t)}{d t} & =\int_{M}\left(\partial_{t} u \cdot \ln u+\partial_{t} u-(m+1) p u \ln u\right) d \mu_{g(t)} \\
& =\int_{M}(-\Delta u \ln u+(m+1) p u) d \mu_{g(t)} \\
& =\int_{M}\left(|\nabla \ln u|^{2}+(m+1) p\right) u d \mu_{g(t)},
\end{aligned}
$$

where we have used the integration by parts to get the last inequality.

It is known that $p \geq 0$ [Fis04], hence $\frac{d \tilde{\mathcal{E}}(t)}{d t} \geq 0$. When $\frac{d \tilde{\mathcal{E}}(t)}{d t}=0$, then $p(t)=0$, by equation (1.1) we have $\operatorname{Rc}_{g(t)}=-m g(t)$.

In the following we will not directly use Theorem A to prove Theorem 1.1. rather we use the method in GPT13 to derive the correct formula for functional $\tilde{\mathcal{W}}$ as given in (1.2) which is not any of the functionals defined in (2.2) and (2.3) when $\alpha$ is defined by CRF (1.1).

Proof of Theorem 1.1, When the abstract flow (2.1) is CRF (1.1), we have $A=(m+1) p$. It follows from [GPT13, Theorem 2.1] that

$$
\frac{d^{2} \tilde{\mathcal{E}}(t)}{d t^{2}}=2 \int_{M}\left(|\mathrm{Rc}+(p+m) g-\nabla \nabla \ln u|^{2}+\Theta(\nabla \ln u)\right) u d \mu_{g}
$$

where

$$
\begin{aligned}
\Theta(\nabla \ln u)= & -(p+m)|\nabla \ln u|^{2}+(m-1)\langle\nabla p, \nabla \ln u\rangle \\
& +\frac{m+1}{2}\left(\partial_{t} p-\Delta p\right)-|\operatorname{Rc}+(p+m) g|^{2} .
\end{aligned}
$$

First we rewrite the following four integrals appeared in (2.8). By integration by parts we have

$$
\begin{aligned}
\int_{M}|\nabla \ln u|^{2} p u d \mu_{g(t)} & =\int_{M}\langle\nabla \ln u, \nabla u\rangle p d \mu_{g(t)} \\
& =-\int_{M}\langle\nabla p, \nabla u\rangle d \mu_{g(t)}-\int_{M} \Delta \ln u \cdot p u d \mu_{g(t)} \\
& =\int_{M} \Delta p \cdot u d \mu_{g(t)}-\int_{M} \Delta \ln u \cdot p u d \mu_{g(t)} .
\end{aligned}
$$


By (2.7) we have

$$
\int_{M}|\nabla \ln u|^{2} u d \mu_{g(t)}=\frac{d \tilde{\mathcal{E}}(t)}{d t}-(m+1) \int_{M} p u d \mu_{g(t)}
$$

We also have

$$
\int_{M}\langle\nabla p, \nabla \ln u\rangle u d \mu_{g(t)}=-\int_{M} \Delta p \cdot u d \mu_{g(t)}
$$

Finally using equation (1.3) and the integration by parts again we have

$$
\begin{aligned}
\int_{M} \partial_{t} p \cdot u d \mu_{g(t)} & =\frac{d}{d t} \int_{M} p u d \mu_{g(t)}-\int_{M} p \cdot \partial_{t} u d \mu_{g(t)}+(m+1) \int_{M} p^{2} u d \mu_{g(t)} \\
& =\frac{d}{d t} \int_{M} p u d \mu_{g(t)}+\int_{M} p \Delta u d \mu_{g(t)} \\
& =\frac{d}{d t} \int_{M} p u d \mu_{g(t)}+\int_{M} u \Delta p d \mu_{g(t)} .
\end{aligned}
$$

Plugging (2.9), (2.10), (2.11), and (2.12) into (2.8), and rearranging terms we get

$$
\begin{aligned}
& \frac{d}{d t} \int_{M}\left(|\nabla \ln u|^{2}+2 m \ln u\right) u d \mu_{g(t)} \\
= & \frac{d^{2} \tilde{\mathcal{E}}(t)}{d t^{2}}+2 m \frac{d \tilde{\mathcal{E}}(t)}{d t}-(m+1) \frac{d}{d t} \int_{M} p u d \mu_{g(t)} \\
= & 2 \int_{M}|\mathrm{Rc}+(p+m) g-\nabla \nabla \ln u|^{2} u d \mu_{g(t)} \\
& -2 \int_{M}|\mathrm{Rc}+(p+m) g|^{2} u d \mu_{g(t)}+2 m(m+1) \int_{M} p u d \mu_{g(t)} \\
& -2 m \int_{M} \Delta p \cdot u d \mu_{g(t)}+2 \int_{M} \Delta \ln u \cdot p u d \mu_{g(t)} .
\end{aligned}
$$

For the further simplification we rewrite the following three expressions in (2.13). Using scalar curvature $R(g(t)) \equiv-m(m+1)$ and CRF (1.1) we have

$$
\begin{aligned}
& |\mathrm{Rc}+(p+m) g-\nabla \nabla \ln u|^{2} \\
= & |\mathrm{Rc}+m g-\nabla \nabla \ln u|^{2}-2 p \Delta \ln u+(m+1) p^{2}, \\
& |\mathrm{Rc}+(p+m) g|^{2}=|\mathrm{Rc}+m g|^{2}+(m+1) p^{2}, \\
& \Delta p=(m+1) p-\frac{1}{m}|\mathrm{Rc}+m g|^{2} .
\end{aligned}
$$


Plugging equations (2.14), (2.15), and (2.16) into (2.13) and simplifying we get

$$
\begin{aligned}
& \frac{d}{d t} \int_{M}\left(|\nabla \ln u|^{2}+2 m \ln u\right) u d \mu_{g(t)} \\
= & 2 \int_{M}|\mathrm{Rc}+m g-\nabla \nabla \ln u|^{2} u d \mu_{g(t)}-2 \int_{M} \Delta \ln u \cdot p u d \mu_{g(t)} .
\end{aligned}
$$

To simplify the last term in (2.17), using integration by parts and equation (2.16) we have

$$
\begin{aligned}
\int_{M} \Delta \ln u \cdot p u d \mu_{g(t)}= & -\int_{M}|\nabla \ln u|^{2} p u d \mu_{g(t)}+\int_{M} \Delta p \cdot u d \mu_{g(t)} \\
= & -\int_{M}|\nabla \ln u|^{2} p u d \mu_{g(t)}+(m+1) \int_{M} p u d \mu_{g(t)} \\
& -\frac{1}{m} \int_{M}|\operatorname{Rc}+m g|^{2} u d \mu_{g(t)} \\
= & -\int_{M}|\nabla \ln u|^{2} p u d \mu_{g(t)}-\int_{M}|\nabla \ln u|^{2} u d \mu_{g(t)} \\
& +\frac{d \tilde{\mathcal{E}}(t)}{d t}-\frac{1}{m} \int_{M}|\operatorname{Rc}+m g|^{2} u d \mu_{g(t)},
\end{aligned}
$$

where we have used equation (2.7) to get the last equality. Now we plug equation (2.18) into (2.17) and obtain

$$
\begin{aligned}
& \frac{d}{d t} \int_{M}\left(|\nabla \ln u|^{2}+2 m \ln u\right) u d \mu_{g(t)} \\
= & 2 \int_{M}|\mathrm{Rc}+m g-\nabla \nabla \ln u|^{2} u d \mu_{g(t)}+2 \int_{M}|\nabla \ln u|^{2} p u d \mu_{g(t)} \\
& +2 \int_{M}|\nabla \ln u|^{2} u d \mu_{g(t)}-2 \frac{d \tilde{\mathcal{E}}(t)}{d t}+\frac{2}{m} \int_{M}|\mathrm{Rc}+m g|^{2} u d \mu_{g(t)} .
\end{aligned}
$$

This proves formula (1.4).

Since pressure function $p \geq 0$, we have $\frac{d \tilde{\mathcal{W}}(t)}{d t} \geq 0$. When $\frac{d \tilde{\mathcal{W}}(t)}{d t}=0$, then each term on the right-hand-side of equation (1.4) equals to zero. In particular, $\mathrm{Rc}=-m g$.

Remark 2.2. Although $\partial_{t} p$ comes up in the proof of the monotonicity of entropy $\tilde{\mathcal{W}}, \tilde{\mathcal{W}}$ itself do not contain any p-term. One way to think about it is that this is about the monotonicity formula for the following flow

$\partial_{t} g=-2\left(\operatorname{Rc}_{g(t)}+\left(\frac{1}{m}\left(-\Delta_{g(t)}+(m+1)\right)^{-1}\left|\mathrm{Rc}_{g(t)}+m g(t)\right|^{2}+m\right) g(t)\right)$.

\section{Another proof of Theorem 1.1 And some Remarks}

Following the idea of [Per02, $\$ 9]$ now we give another proof of Theorem 1.1. Let $u=e^{-f}$ be a positive solution of (1.3) on closed manifold $M^{m+1}$, 
then

$$
\partial_{t} f+\Delta f-|\nabla f|^{2}+(m+1) p=0 .
$$

Note that we can rewrite the entropy

$$
\begin{aligned}
\tilde{\mathcal{W}}\left(g, e^{-f}\right) & =\int_{M}\left(|\nabla f|^{2}-2(m+1) f\right) e^{-f} d \mu_{g} \\
& =\int_{M}\left(2 \Delta f-|\nabla f|^{2}-2(m+1) f\right) e^{-f} d \mu_{g} .
\end{aligned}
$$

We will compute the evolution equation of

$$
v=\left(2 \Delta f-|\nabla f|^{2}-2(m+1) f\right) e^{-f}
$$

using backward parabolic operator $\square^{*}=-\partial_{t}-\Delta_{g(t)}+(m+1) p$.

Let

$$
w=2 \Delta f-|\nabla f|^{2}-2(m+1) f .
$$

We compute

$$
\begin{aligned}
\square^{*} w= & -2\left(\partial_{t}+\Delta\right) \Delta f+\left(\partial_{t}+\Delta\right)|\nabla f|^{2}+2(m+1) p \Delta f \\
& -(m+1) p|\nabla f|^{2}+2(m+1)\left(\partial_{t}+\Delta-(m+1) p\right) f \\
= & -2 \Delta\left(\partial_{t}+\Delta\right) f-4 R_{i j} \nabla_{i} \nabla_{j} f-4(p+m) \Delta f-4 \nabla_{i} p \nabla_{i} f \\
& +4 R_{i j} \nabla_{i} f \nabla_{j} f+2|\nabla \nabla f|^{2}+2(p+m)|\nabla f|^{2}+2 \nabla_{i}|\nabla f|^{2} \nabla_{i} f \\
& +2(m+1) p \Delta f-(m+1) p|\nabla f|^{2} \\
& +2(m+1)\left(|\nabla f|^{2}-(m+1) p-(m+1) p f\right),
\end{aligned}
$$

where to get the last equality we have used

$$
\partial_{t} \Delta f=\Delta \partial_{t} f+2 R_{i j} \nabla_{i} \nabla_{j} f+2(p+m) \Delta f-(m-1) \nabla_{i} p \nabla_{i} f
$$

(see [CLN06, (S.5) on p.547]) and the parabolic Bochner formula

$$
\begin{aligned}
\left(\partial_{t}+\Delta\right)|\nabla f|^{2}= & 4 R_{i j} \nabla_{i} f \nabla_{j} f+2|\nabla \nabla f|^{2}+2(p+m)|\nabla f|^{2} \\
& +2 \nabla_{i}|\nabla f|^{2} \nabla_{i} f-2(m+1) \nabla_{i} p \nabla_{i} f .
\end{aligned}
$$

Using

$$
\begin{aligned}
-2 \Delta\left(\partial_{t}+\Delta\right) f= & -2 \Delta|\nabla f|^{2}+2(m+1) \Delta p \\
= & -4 R_{i j} \nabla_{i} f \nabla_{j} f-4|\nabla \nabla f|^{2}-4 \nabla_{i} \Delta f \nabla_{i} f \\
& +2(m+1)^{2} p-\frac{2(m+1)}{m}|\mathrm{Rc}+m g|^{2}
\end{aligned}
$$

and by some simplification we get

$$
\begin{aligned}
\square^{*} w= & -2|\mathrm{Rc}+m g+\nabla \nabla f|^{2}-\frac{2}{m}|\mathrm{Rc}+m g|^{2}-2 \nabla_{i} f \nabla_{i} w-2|\nabla f|^{2} \\
& +(m-1) p w-4 \nabla_{i} p \nabla_{i} f-4(m+1) p f .
\end{aligned}
$$


Now we compute

$$
\begin{aligned}
& \square^{*} v=\square^{*}(w u)=\square^{*}\left(w e^{-f}\right) \\
= & \left(\square^{*} w\right) u+w\left(\square^{*} u\right)-2 \nabla w \nabla u-(m+1) p w u \\
= & -2|\operatorname{Rc}+m g+\nabla \nabla f|^{2} u-\frac{2}{m}|\mathrm{Rc}+m g|^{2} u \\
& -2|\nabla f|^{2} u-4 \nabla_{i} p \nabla_{i} f u-4(m+1) p f u-2 p w u \\
= & -2|\operatorname{Rc}+m g+\nabla \nabla f|^{2} u-\frac{2}{m}|\operatorname{Rc}+m g|^{2} u \\
& -2|\nabla f|^{2} u-2|\nabla f|^{2} p u+4 \operatorname{div}(p \nabla u) .
\end{aligned}
$$

The formula (1.4) follows from (3.2) easily, we omit the detail.

Remark 3.1. Because of the term $\operatorname{div}(p \nabla u)$ in formula (3.2) we are not able to prove a pointwise Harnack inequality for heat kernel function $u$ as given in [Per02, Corollary 9.3] and/or [Ni04, Theorem 1.2].

On a closed manifold $M^{m+1}$ we define functional

$$
\tilde{\nu}(g)=\inf _{\int_{M} e^{-f} d \mu_{g}=1} \tilde{\mathcal{W}}\left(g, e^{-f}\right)
$$

for each Riemannian metric $g$. To see that $\tilde{\nu}(g)$ is a finite number, recall that the log-Sobolev inequality for $(M, g)$ says ([Per02, §3], [Ni04, (1.8)]) that there is a constant $c_{1}=c_{1}(M, g)$ such that

$$
\int_{M}\left(|\nabla f|^{2}+f\right) e^{-f} d \mu_{g} \geq c_{1}
$$

for $f$ satisfying $\int_{M} e^{-f} d \mu_{g}=1$. Since $x e^{-x} \leq e^{-1}$ for any real $x$, we have

$$
\begin{aligned}
\tilde{\mathcal{W}}\left(g, e^{-f}\right) & =\int_{M}\left(|\nabla f|^{2}+f\right) e^{-f} d \mu_{g}-(2 m+3) \int_{M} f e^{-f} d \mu_{g} \\
& \geq c_{1}-(2 m+3) e^{-1} \operatorname{vol}(M, g) .
\end{aligned}
$$

An easy consequence the monotonicity formula (1.4) is

Lemma 3.2. $\tilde{\nu}(g(t))$ is monotone increasing under CRF (1.1).

Proof. This can be proved as the well-known proof in [Per02, §2.2].

Remark 3.3. It is not clear to us whether the lower bound $c_{1}$ in 3.4 depends on $c_{2}$ only assuming that $c_{2}$ is the lower bound in $\tilde{\mathcal{W}}\left(g, e^{-f}\right) \geq c_{2}$ for $\int_{M} e^{-f} d \mu_{g}=1$. If it is true then we will have the volume $\kappa$-noncollapsing on scale $r=1$ for CRF $g(t)$.

Remark 3.4. A naive generalization to CRF of the monotonicity of the reduced volume as in Mul10, Theorem 1.4] does not hold since the nonnegative condition Mul10, (1.6)] is false for CRF. 


\section{REFERENCES}

[CLN06] B. Chow, P. Lu, and L. Ni, Hamilton's Ricci flow. Graduate Studies in Mathematics, volume 77, American Mathematical Society, 2006.

[EKNT08] K. Ecker, D. Knopf, L. Ni, and P. Topping, Local monotonicity and mean value formulas for evolving Riemannian manifolds. J. Reine Angew. Math. 616 (2008), 89130.

[Fis04] A. Fischer, An introduction to conformal Ricci flow. Class. Quantum Grav. 21 (2004), 171-218.

[GPT13] H.-X. Guo, R. Philipowski, and A. Thalmaier, Entropy and lowest eigenvalue on evolving manifolds. Pacific J. Math. 264 (2013), 61-81.

[Hui90] G. Huisken, Asymptotic behavior for singularities of the mean curvature flow. J. Diff. Geom. 31 (1990), 285-299.

[Lis08] B. List, Evolution of an extended Ricci flow system. Comm. Anal. Geom. 16 (2008), 1007-1048.

[LQZ14] P. Lu, J. Qing, Y. Zheng, A note on the conformal Ricci flow. Pacific J. Math. 268 (2014), 413-434.

[LQZ19] P. Lu, J. Qing, Y. Zheng, Conformal Ricci flow on asymptotically hyperbolic manifolds. Science China Mathematics, 62 (2019), 157-170.

[Mul10] R. Müller, Monotone volume formulas for geometric flows. J. Reine Angew. Math. 643 (2010), 3-57.

[Mul12] R. Müller, Ricci flow coupled with harmonic map flow. Ann. Sci. Éc. Norm. Supér (4) 45 (2012), 101-142.

[Ni04] L. Ni, The entropy formula for linear heat equation. J. Geom. Anal. 14 (2004), $87-100$.

[Per02] G. Perelman, The entropy formula for the Ricci flow and its geometric applications. arXiv:math.DG/0211159 (2002).

[SZ18] X.-M. Sun and A.-Q. Zhu, Backward uniqueness for the conformal Ricci flow. Diff. Geom. Appl. 56 (2018), 110-119.

[Wa19] L.-F. Wang, Monotonicity of eigenvalues and functionals along the RicciBourguignon flow. J. Geom. Anal., 29 (2019), 1116-1135.

School of Mathematical Sciences, East China Normal University, 500 Dongchuan Road, Shanghai 200241, P. R. of China

E-mail address: lianyisky@163.com

Department of Mathematics, University of Oregon, Eugene, OR 97403, USA

E-mail address: penglu@uoregon.edu

School of Mathematical Sciences, East China Normal University, 500 Dongchuan

Road, Shanghai 200241, P. R. OF China

E-mail address: jianhongwang@163.com

School of Mathematical Sciences, East China Normal University, 500 Dongchuan

Road, Shanghai 200241, P. R. of China

E-mail address: zhyu@math.ecnu.edu.cn 